\title{
Physiological Cost and Thermal Envelope: a Novel Approach to Cycle Garment Evaluation during a Representative Protocol
}

\section{Original Investigation}

\section{Running Head: Evaluating cycle clothing during exercise}

Jo Corbett (Corresponding author)

Department of Sport and Exercise Sciences

University of Portsmouth

Spinnaker Building

Cambridge Road

Portsmouth

PO1 2ER

Tel: +44(0)2392843084 Fax: +44(0)02392843620 E-mail: jo.corbett@ port.ac.uk

Martin J. Barwood

Department of Sport and Exercise Sciences

Northumbria University

E-mail: martin.barwood@northumbria.ac.uk

Michael J. Tipton

Department of Sport and Exercise Sciences

University of Portsmouth

E-mail: michael.tipton@port.ac.uk 


\begin{abstract}
Aim: To examine thermoregulation in different clothing assemblies during a representative cycling exercise protocol. Method: Six males undertook cycling exercise simulating representative thermal exchange challenges, whilst wearing low (LOW), intermediate (INT1 and INT2), or high (HI), amounts of clothing. Exercise was conducted at $14.5^{\circ} \mathrm{C}, 46.8 \%$. R.H., and included a 'flat' (45 minutes at 35\% peak power output (PPO), wind speed $8.3 \mathrm{~m} \cdot \mathrm{s}^{\text {' }}$ ${ }^{1}$ ); 'uphill' (30 minutes at 55\% PPO, wind speed $3.6 \mathrm{~m} \cdot \mathrm{s}^{-1}$ ), and 'downhill' (20 minutes at 50 $\mathrm{W}$, wind speed $16.7 \mathrm{~m} \cdot \mathrm{s}^{-1}$ ) stage. Results: Rectal temperature changed with the exercise stage and was independent of clothing assembly. In contrast, an 'envelope' was evident for mean body temperature, resulting from differences in mean skin temperature between the LOW and HI conditions. The elevated mean body temperature in HI was associated with increased physiological 'cost', in the form of increased sweat production and heart rate. Conclusion: Physiological 'cost' provides a better index of clothing performance than deep body temperature in the 'thermoregulatory zone', as a consequence sports clothing should attempt to optimise the balance between comfort and reduced 'physiological cost.'
\end{abstract}

Key Words: Environmental physiology, clothing, temperature 


\section{Introduction}

During cycling exercise metabolic heat production varies mainly as a function of power output, which is typically stochastic due to terrain and tactical influences (Jeukendrup, 2002; Vogt et al., 2007). Simultaneously, the avenues for biophysical heat exchange may be affected: alterations in cycling speed will influence convective cooling, as well as evaporative cooling, through effects on the boundary layer around the body. Consequently, multiple thermal exchange scenarios can arise in a single cycling event. For example, during downhill cycling, metabolic heat production may be low (Vogt et al., 2007), whereas convective and evaporative heat losses can increase due to elevated airflow (Saunders et al., 2005). In uphill cycling, metabolic heat production is typically increased (Vogt et al., 2007), whilst lower airflow will decrease heat loss by convection and evaporation (Saunders et al., 2005). This variety of thermal exchange scenarios can make the selection of appropriate clothing for a given cycling event problematic, particularly when opportunities for adding or removing clothing are limited, such as during competition.

Clothing affects biophysical heat exchange by increasing insulation and acting as a barrier to convective and evaporative heat exchange (Berglund \& Gonzalez, 1977; Gavin, 2003; Nagata, 1978). However, excessive clothing will lead to increased sweating in an attempt to regulate body temperature. If the clothing acts as a barrier to evaporation this may cause skin wettedness, discomfort (Pascoe et al., 1994), and increased thermal stress (Brownlie et al., 1987); the cooling efficiency of sweating is also reduced when moisture is wicked away from the skin before it evaporates (Havenith et al., 2013). Thus, the ideal clothing for preventing heat loss is that which blocks air movement, but allows the evaporation of water vapour from the skin if sweating occurs (Gavin, 2003). In contrast, clothing for facilitating heat loss 
should have minimal insulation and pose little resistance to the evaporation of sweat from the skin or convective airflow (Gavin, 2003), whilst clothing that reflects solar radiation will reduce heat gain from the environment (Nielsen, 1990).

Although manikins can measure heat flux, it is often difficult to accurately replicate the friction, drag, and cling between layers and against skin that is encountered during exercise, as well as the subsequent impact on the microclimate between the skin and clothes (Pascoe et al., 1994). Manikins also lack the important associated thermoregulatory and perceptual responses. It has also previously been noted that research examining the influence of clothing on thermoregulation has generally failed to investigate high exercise intensities (Gavin, 2003), to mimic the airflow of outdoor conditions (Gavin, 2003), or to employ prolonged and ecologically valid sports-related exercise protocols (Davis \& Bishop, 2013). This is particularly pertinent with regard cycle clothing, which is often used during high intensity exercise and in conditions with high airflow rates. To our knowledge, no previous studies have attempted to simulate a range of different thermal exchange scenarios in a single protocol, in order to evaluate cycle garment performance in an ecologically relevant manner.

Finally, attempts to evaluate thermal strain during exercise have often focused on changes in deep body temperature as the primary index of physiologic stress (Moran et al., 1998). Whilst this may have historical precedent (Haldane, 1905) and is likely practical under conditions of un-compensable heat stress (Gonzalez et al., 1997), the equilibrated deep body temperature of an exercising human is independent of ambient conditions across a wide range of environments and is determined by the external work rate (Nielsen, 1938). Sustained exercise at work rates and environment combinations that do not allow thermal balance (i.e. outside the "thermoregulatory zone" (Tipton, 2006)) result in an inexorable rise in deep body 
temperature until the attainment of critical values associated with exercise termination (González-Alsonso et al., 1999). Thus, during prolonged exercise athletes typically select work rates that allow thermal compensability in their environment (Schlader et al., 2011), that is they remain within the "thermoregulatory zone". In such situations the efficacy of deep body temperature as a measure of physiologic strain is limited; this includes many ecologically valid exercise scenarios. It has long been known that even under thermally compensable conditions thermo-effector responses, such as heart rate, peripheral blood flow, and sweating are elevated as thermal stress is increased, in advance of uncompensable alterations in deep body temperature (Lind, 1963); this 'physiological cost' can provide an index of the physiological strain.

Accordingly, the aim of the present study was to examine the thermoregulatory and perceptual responses of trained cyclists, during a representative cycle exercise protocol simulating different thermal exchange scenarios, whilst wearing a range of different cycle clothing assemblies. We hypothesised that the 'physiological cost' associated with cycle clothing when undertaking an ecologically valid cycle test within the thermoregulatory zone would provide a useful index of clothing performance. 


\section{Method}

\section{Participants}

Six male trained-cyclists volunteered to participate and provided their written informed consent. Inclusion criteria were: a satisfactory medical examination including: a 12 lead electrocardiogram: a satisfactory health history questionnaire, with no contra-indications for maximal exercise; and, a peak power output $>300 \mathrm{~W}$ during an initial incremental ramp exercise test. The mean (SD) age, height, mass, peak power output (PPO) and maximum rate of oxygen uptake $\left(\mathrm{VO}_{2 \max }\right)$ of the participants were: $29(9)$ years, $1.79(0.10) \mathrm{m}, 74.06(8.27)$ $\mathrm{kg}, 400(51) \mathrm{W}$, and $59.1(4.3) \mathrm{mL} \cdot \mathrm{kg}^{-1} \cdot \mathrm{min}^{-1}$, respectively. The study was approved by the University’s Biosciences Research Ethics Committee.

\section{Experimental Design}

Following a preliminary incremental exercise trial for the determination of $\mathrm{PPO}$ and $\mathrm{VO}_{2 \max }$, participants undertook four experimental trials wearing a different clothing assembly on each occasion. The order in which participants undertook the experimental trials was determined using a Latin-square. Each participant undertook their exercise trials at the same time of day ( \pm 1 hour), with at least 48 hours between tests.

\section{Experimental Procedure}

The preliminary incremental exercise test took place under ambient laboratory conditions, with a fan available to provide cooling if requested by the participant. The participant commenced exercise at a power output of $60 \mathrm{~W}$, which was increased by $30 \mathrm{~W} \cdot \mathrm{min}^{-1}$ until the participant could no longer maintain the required power output. The final power achieved at 
the end of the exercise test was termed the PPO, with the highest $\mathrm{VO}_{2}$ obtained from a Douglas bag collection $>15$ seconds termed the $\mathrm{VO}_{2 \max }$.

The experimental trials took place in an environmental chamber at an ambient temperature of $14.5(0.2)^{\circ} \mathrm{C}$ and relative humidity of 46.8 (2.9)\%. During each trial participants undertook an exercise protocol lasting $\sim 2$ hours, which was designed to simulate a range of representative thermal exchange scenarios. The protocol consisted of: i) 45 minutes moderate intensity exercise (35\% PPO) with a moderate air speed $\left(8.3 \mathrm{~m} \cdot \mathrm{s}^{-1}\right)$, simulating a 'flat' cycling thermal exchange scenario; ii) 30 minutes high intensity exercise (55\% PPO) with a low air speed $\left(3.6 \mathrm{~m} \cdot \mathrm{s}^{-1}\right)$, simulating an 'uphill' cycling thermal exchange scenario; iii) 5 minutes rest period with no wind, simulating a brief rest period; iv) 20 minutes low intensity exercise (50 W) with a high air speed $\left(16.7 \mathrm{~m} \cdot \mathrm{s}^{-1}\right)$, simulating a 'downhill' cycling thermal exchange scenario; v) 5 minutes rest period with no wind; vi) $10 \mathrm{~km}$ self-paced time-trial with a moderate air speed $\left(8.3 \mathrm{~m} \cdot \mathrm{s}^{-1}\right)$, to obtain an index of performance.

All exercise trials took place on the participant's own bicycle, mounted on a Computrainer (Racermate, Seattle, USA) ergometer calibrated in accordance with previously described methods (Davison et al. 2009). During the $10 \mathrm{~km}$ time-trial participants completed a virtual race-course constructed using the Computrainer 3D software (Racermate, Seattle USA). The distance completed was displayed throughout the time-trials, but all other feedback was blinded to the participant. Wind was simulated by use of a $0.5 \mathrm{~m}$ industrial fan (Fläktwoods, Colchester, UK) positioned at a height of $1.15 \mathrm{~m}$ (to centre point of fan) and $0.50 \mathrm{~m}$ from the centre point of the front wheel of the participant's bicycle. Air speed was measured at a height of $1.40 \mathrm{~m}$ and distance of $0.60 \mathrm{~m}$ from the fan using an anemometer (Meterman TMA10, Wavetek, San Diego, USA). 
On each occasion during the experimental tests participants wore one of the following clothing assemblies: i) Low clothing (LOW) consisting of cycling shorts (74\% nylon, 26\% elastane); ii) Intermediate clothing 1 (INT1) consisting of bib style cycling shorts (78\% nylon, $22 \%$ elastane; mesh $82 \%$ polyester, $18 \%$ elastane) and a short sleeve cycling jersey (100\% polyester; mesh 94\% nylon, 6\% elastane); iii) Intermediate clothing 2 (INT2) consisting of a different set of bib style cycling shorts (75\% polyamide, $22 \%$ elastane, $3 \%$ carbon fibre) and different short sleeve cycling jersey (54\% polyamide, $36 \%$ polyester, $7 \%$ elastane, $3 \%$ lyocell), iv) High clothing (HI) consisting of INT1 plus a long sleeve base layer (84\% polyester, $16 \%$ elastane), cycling trousers (100\% nylon, polytetrafluoroethylene membrane; insert $94 \%$ nylon, $6 \%$ elastane), cycling jacket (100\% nylon, polytetrafluoroethylene membrane; insert 94\% nylon, $6 \%$ elastane), skull cap (100\% polyester polytetrafluoroethylene membrane; insert $87 \%$ nylon, $13 \%$ elastane). In all of the conditions the participants also wore the same fingerless gloves, socks, cycling helmet, and cycling shoes. Clothing was fitted in accordance with manufacturers' guidelines and the LOW and HI conditions were selected to represent the upper and lower boundaries of clothing assembly that might be worn under the exercise conditions examined. This was done deliberately to create a reference "envelope" within which to gauge the performance of the other garments.

\section{Measurements}

Preliminary Incremental Exercise Trial

During the preliminary test, power output was controlled using the Computrainer control panel and consecutive 60 second expired gas collections were obtained using the Douglas bag method. Expired gases were analysed to determine gas fractions and volumes using a gas 
analyser (Servomex PLC., Crowborough, UK) calibrated using gases of known concentration and dry gas meter (Harvard Apparatus Ltd. , Edenbridge, UK), respectively.

\section{Experimental Trials}

Deep body temperature was measured using a thermistor (Grant Instruments, Cambridge, UK) self-inserted in the rectum, $15 \mathrm{~cm}$ beyond the anal sphincter (Squirrel Data Logger, Grant Instruments Ltd, Cambridge, UK). Skin temperature $\left(T_{\mathrm{sk}}\right)$ was measured using thermistors (DS18B20 T-3, MSR, Switzerland) attached to the skin by a single piece of Tegaderm $^{\mathrm{TM}}$ tape at six sites on the right side of the participant at: chest, back, bicep, forearm, thigh, calf. Deep body temperature and skin temperatures were recorded every 60 seconds throughout experimental trials using an MSR data logger (MSR12, MSR electronics GmbH, Henggart, Switzerland). Ambient conditions were measured using a WBGT weather station (Grant Instruments, Cambridge, UK). Mean body temperature was determined according to Colin et al. (1971) and mean skin temperature using Ramanathan (1964). Throughout the experimental trial participants wore a heart rate monitor (Polar RS800, Oy, Finland), which recorded heart rate every 15 seconds. Fluid intake and pre and post naked (following towel drying) and clothed body weights (OHAUS I-10 digital scales, Canada) were used to estimate total sweat production. Local sweat rate was determined using a ventilated capsule positioned on the forehead under the cycling helmet (Q-Sweat Quantitative Sweat Measurement System, Model 1.0, WR Medical Electornics, Co., Mn, USA). To provide an index of peripheral blood flow a laser Doppler probe (Moor Instruments, Axeminster, UK) was lightly taped to the right index finger, with data corrected against a biological zero taken at the start of each trial by inflating a blood pressure cuff to $250 \mathrm{mmHg}$ to momentarily occlude the brachial artery. Five minutes before the end of each exercise stage of the experimental trial a 60 second expired gas collection was obtained and analysed 
to determine the rate of oxygen uptake $\left(\mathrm{VO}_{2}\right)$. Rating of perceived exertion (RPE (Borg 1982)) and perceptual measures of thermal comfort and thermal sensation (Zhang, 2003) were obtained at 5 minute intervals throughout the trials.

\section{Data analyses}

Data are presented as mean (SD) unless otherwise stated. Where data are available at 60 second intervals (i.e. deep body temperature, mean body temperature, mean skin temperature, heart rate) they were averaged for each participant over the final five minutes of each of the exercise stages of the experimental protocol i.e. final five minutes of the 45 minute 'flat' stage, final five minutes of the 30 minute 'uphill' stage, final five minutes of the 20 minute 'downhill' stage for comparisons. Where data are reported at 5 minute intervals (i.e. RPE, thermal comfort, thermal sensation) the final data point recorded for each exercise stage was used for analysis. Between groups' differences where determined by Freidman's test, with significant differences examined post-hoc using the Wilcoxon signed ranks test. The alpha value was set a priori at 0.05 . 


\section{Results}

\section{Thermal measures}

There were no significant differences between clothing conditions in rectal temperature at any of the comparison points throughout the exercise trials (Figure 1a). In general rectal temperature rose towards a plateau throughout the first 45 minutes of exercise, with a further increase occurring during the subsequent 30 minute uphill exercise stage. During the 20 minute simulated downhill cycling stage a reduction in rectal temperature was observed. In contrast, significant differences in mean skin temperature were evident between all of the clothing assemblies at comparison point 1 and comparison point 3 . At the second comparison point mean skin temperature was not different between INT1vs. INT2 and LOW vs. INT2, but was different between all other conditions (Figure 1b). Significant differences in mean body temperature were evident at comparison point 1 and comparison point 3 between all conditions except INT1 vs. INT2. At comparison point 2 differences in mean body temperature were evident between all conditions except INT1 vs. INT2 and LOW vs. INT2 (Figure 1c). Across the experimental protocol a clear thermal 'envelope' was evident for mean skin temperature and mean body temperature, the upper and lower boundaries of which were defined by the HI and LOW clothing conditions, respectively

Site specific chest and back temperature data are presented together in figure $1 \mathrm{~d}$, with significant differences between conditions denoted accordingly. In the main, chest temperature was different between all conditions, with INT1 being lower than INT 2, although both were positioned within the boundaries of the thermal 'envelope' defined by HI and LOW. Back temperatures were similar between INT1 and INT2 across the exercise protocol, and a clear thermal envelope was defined by the HI and LOW conditions. However, 
back temperature in INT1 and INT2 approached the upper end of the thermal envelope at the second comparison point.

\section{Physiological measures}

A significant effect of clothing condition on heart rate was evident at the second comparison point, with heart rate being higher in the HI condition than in either the LOW condition or INT2 (figure 2). Between conditions differences in heart rate at the other comparisons points were not statistically significant. Significant differences in forehead sweat rate were evident between LOW vs. HI and HI vs. INT1 at comparison point 1 (figure 3). Sweat rate was increased at the second comparison point, but was not different between conditions, suggesting that the peak local sweating rate had been achieved in all conditions. At the third comparison point differences in sweat rate were evidence between each condition with the exception of INT1 vs. INT2 and LOW vs. INT1. Total sweat production was $0.35(0.06) \mathrm{L} \cdot \mathrm{hr}^{-}$ 1, 0.43(0.05) $\mathrm{L} \cdot \mathrm{hr}^{-1}, 0.42(0.06) \mathrm{L} \cdot \mathrm{hr}^{-1}, 0.71(0.06) \mathrm{L}^{-\mathrm{hr}^{-1}}$ for LOW, INT1, INT2, and HI, respectively, and was different between all clothing conditions with the exception of INT1 and INT2. In contrast, no significant differences were evident in the rate of oxygen uptake, or fingertip blood flow, at any of the comparison points. Mean power output sustained during the $10 \mathrm{~km}$ performance trial was 272(15) W, 265(18) W, 266(20) W 259(27) W for LOW, INT1, INT2, and HI, respectively, and was not significantly different between clothing conditions.

\section{Perceptual measures}

The rating of perceived exertion was not different between the clothing conditions during the first two comparison points, but was significantly higher in INT2 than LOW at the third comparison point. Thermal sensation data indicated that participants felt significantly warmer 
in HI than in LOW, INT1 or INT2 at the first comparison point. At the second comparison point participants felt significantly hotter in HI than in LOW or INT2, and also in INT1 and INT2 than in LOW. At the third comparison point participants felt significantly warmer in HI than in LOW, INT1, or INT2. Finally, there were no significant between conditions differences in thermal comfort at the first or third comparison point. Although participants felt significantly less comfortable in HI than in LOW, INT1 or INT2 at the second comparison point. 


\section{Discussion}

The ultimate validation of any clothing assembly relies on testing with humans under the actual conditions of intended use (Pascoe et al., 1994). The present study is the first to employ a prolonged and ecologically valid sport-related exercise protocol to evaluate the performance of different cycle garment assemblies during exercise in a range of representative thermal exchange scenarios which mimic the airflow of outdoor conditions. In all clothing assembly conditions the rectal temperature response was independent of the clothing worn. Rectal temperature increased throughout the 'flat' cycling exercise stage, with a further increase during the 'uphill' exercise stage, prior to a reduction during the 'downhill' exercise stage. This finding is consistent with other studies which have shown that the temperature at which deep-body temperature is regulated during exercise is primarily related to the relative exercise intensity (Gant et al., 2004; Lind, 1963; Nielsen, 1938; Saltin \& Hermansen, 1966). In contrast, mean skin temperature, and consequently mean body temperature, was influenced by the clothing assembly. In each instance the mean skin and mean body temperature was highest in the HI condition and lowest in LOW, with INT1 and INT2 positioned towards the lower end of the thermal 'envelope.' Since work output was identical between each of the exercise conditions (except during the TT), these differences must result solely from alterations in heat loss capacity as a consequence of the different clothing assemblies. The boundaries of the thermal 'envelope' defined by the LOW and HI condition should be a useful reference for clothing manufacturers by providing an indication of the realistic limits for heat loss and heat retention that are possible for each exercise stage via alterations in garment assembly selection, or design.

The site specific temperature data from the chest and back are useful in illustrating the nonuniform nature of the torso skin temperature during cycling exercise in an environment where 
a representative air velocity is provided. Although not examined statistically, an elevated back temperature relative to chest temperature was particularly evident at the higher air velocities in INT1, INT2 and LOW conditions (comparison point 1 and 3). In addition, it is interesting to note that the back temperature in INT1 and INT2 conditions approached the upper limit of the thermal 'envelope' for back temperature (e.g. HI condition) at the second comparison point. We believe that these site specific data are useful for two reasons: firstly, they demonstrate the importance of using a representative air velocity when examining the performance of sporting garments; failure to do so will lead to an inaccurate representation of the skin:clothing microclimate during exercise, and will diminish heat loss to the environment (Saunders et al., 2005). Secondly, the observation that back temperature in the INT1 and INT2 conditions approached the upper (HI) limit of the thermal 'envelope' at the second comparison point, implies that increased back ventilation may be a beneficial design feature for these assemblies in this thermal exchange scenario.

Sweat production was influenced by the clothing assembly worn, and differences in local sweat rates were evident at the first and third comparison point. However, local sweat rates were not different between conditions at the second comparison point, possibly indicating that the peak local sweating rate had been reached. Nevertheless, heart rate was elevated in HI relative to INT2 and LOW at the second comparison point. Presumably, these augmented effector responses with increased amounts of clothing occurred as a consequence of the elevated mean skin temperature and mean body temperature, in order to facilitate heat loss, and as such, demonstrate the increased physiological 'cost' associated with regulating deep body temperature whilst wearing different garment assemblies. It is well established that sweat losses of $\sim 2 \%$ body weight can result in significant impairments in performance (Cheuvront et al., 2009), whilst an elevated heart rate during sub-maximal exercise at a fixed 
intensity is indicative of increased physiological strain. However, there was no significant difference in $10 \mathrm{~km}$ TT performance between the clothing conditions in the present investigation, although, numerically, the trends for performance times were consistent with the differences in mean body and mean skin temperature. This indicates that the increased physiological cost was tolerated by the trained cyclists participating in this study, although the participant numbers used in the present study will have impact upon the ability to detect a statistically significant difference; it remains to be confirmed whether a statistically significant effect would have been evident with a larger number of participants, or if the increased physiological cost would have been tolerated by less well trained participants, or during exercise in a warmer exercise environment. Nevertheless, it would appear logical to suggest that garments intended for use in sports performance should be designed in order to achieve the best thermal response at minimum "physiological cost". In most prolonged exercise activities, during which participants must remain in the thermoregulatory zone (i.e. able to thermoregulate), it will be the physiological cost of thermoregulating, rather than body temperature per se which will be the most illuminating measure of clothing performance.

Differences in thermal sensation between clothing conditions were evident at each of the comparison points, indicating that the clothing assemblies were sufficiently different to induce significant perceptual differences. In contrast, there was no difference in thermal comfort between conditions at the first and third comparison points, although thermal comfort was significantly lower in the HI condition than in the other conditions at the end of the uphill exercise stage. The observation that participants generally remained comfortable and that there were no between conditions differences in thermal comfort during the first and third stage suggests that the range of clothing assemblies selected were realistic under these 
conditions. Taken together these findings emphasise the complex nature of thermal comfort perception, which may remain relatively constant (comparison point one and three) in the presence of a variety of whole-body thermal sensations. Interestingly, the RPE did not differ between groups at the point at which a significant difference in thermal comfort was detected. This would imply that thermal comfort is not an important factor determining perception of exertion, or that that there is a lag in the relationship between thermal comfort and RPE. It should be noted that RPE was higher in INT2 than LOW at the third comparison point, although we question the practical significance of this finding. Further research is required to understand the nature of the factors influencing thermal and effort perception under the nonuniform and dynamic exercise conditions employed in the present study, and frequently encountered outside of the laboratory environment.

In summary, the representative cycle exercise protocol employed in this study enabled cycle garment performance to be evaluated across a range of different thermal scenarios, in a single exercise test. Rectal temperature remained in the thermoregulatory zone and changed concomitant with the intensity of exercise and was independent of the clothing assembly. In contrast, a clear 'thermal envelope' was evident for mean body temperature, which resulted from differences in mean skin temperature as a consequence of the various garment assemblies used. Thus, future studies examining clothing performance during exercise within the thermoregulatory zone should not use deep body temperature as the sole index of thermal performance. The elevated mean body temperature with increased levels of clothing resulted in an elevated rate of sweat production and heart rate at points during the exercise trials. Thus, regulating deep body temperature within the thermoregulatory zone under different clothing conditions is associated with varying physiological 'cost'. This physiological 'cost' may have the potential to impair performance, although this was not apparent in the present 
study. We propose that the thermal 'envelope' and physiological 'cost' of thermoregulating within the thermoregulatory zone represent useful concepts for evaluating the performance of sporting apparel. Finally, laboratory studies examining the performance of sporting apparel should seek to accurately replicate the range of metabolic and thermal demands likely to be encountered by the end-user. 


\section{Perspective}

There have been calls for studies examining the influence of clothing on thermoregulation to employ prolonged, ecologically relevant, sports-related exercise protocols including high exercise intensities and mimicking the airflow of outdoor conditions (Gavin, 2003; Davis \& Bishop, 2013). This study used a novel protocol to examine thermoregulatory and physiological responses during prolonged cycling exercise in representative thermalexchange challenges, with different cycle-clothing assemblies; the concepts of "physiological cost" and "thermal "envelope" have been introduced. During most prolonged exercise activities athletes remain in the "thermoregulatory zone"; we proposed the magnitude of thermoeffector responses necessary to maintain the regulated deep body temperature (i.e. "physiological cost"), rather than body temperature per se, as an index of clothing performance. Differences in "physiological cost" were evident between clothing assemblies in the absence of change in deep body temperature. "Thermal envelope" refers to boundary thermal profiles elicited by clothing assemblies towards the upper and lower end of those that might be worn under given environmental conditions. This "envelope" in mean body temperature was demonstrated in this study and might represent a useful approach for providing an indication of the realistic limits for heat loss and heat retention possible via alterations in garment assembly selection or design. 


\section{Acknowledgements}

The authors would like to acknowledge the technical assistance provided by Geoff Long, Alex Ouzounoglou, and Nicola Ferguson. 


\section{References}

Berglund LG, Gonzalez RR. Evaporation of sweat from sedentary man in humid environments. J Appl Physiol 1977: 42(5): 767-772.

Brownlie L, Mekjavic I, Bannister E. Thermoregulation in athletic racing apparel. Ann Physiol Anthropol 1987: 6(3) 145-155.

Borg GA. Psychophysical bases of perceived exertion. Med Sci Sports Exerc 1982: 14(5): $377-381$.

Cheuvront SN, Carter R, Sawka MN. Fluid balance and endurance exercise performance. Curr Sports Med Rep 2003: 2(4): 202-208.

Colin J, Timbal J, Houdas Y, Boutelier C, Guieu JD. Computation of mean body temperature from rectal and skin temperatures. J Appl Physiol 1971: 31(3): 484-489.

Davis JK, Bishop PA. Impact of clothing on exercise in the heat. Sports Med 2013, [E-pub ahead of print], DOI 10.1007/s40279-013-0047-8.

Davison RCR, Corbett J, Ansley L. Influence of temperature and protocol on the calibration of the Computrainer electromagnetically-braked cycling ergometer. International SportsMed Journal 2009: 10: 66-76.

Gant N, Williams C, King J, Hodge BJ. Thermoregualtory responses to exercise: relative versus absolute intensity. J Sports Sci 2004: 22 (11-12): 1083-1090. 
Gavin TP. Clothing and thermoregulation during exercise. Sports Med 2003: 33(13): 941947.

Gonzalez RR, McLellan TM, Withey WR, Chang SK, Pandolf KB. Heat strain models applicable for protective clothing systems: comparison of core temperature response. $J$ Appl Physiol 1997: 83(3): 1017-1032.

González- Alonso J, Teller C, Andersen SL, Jensen FB, Hyldig T, Nielsen B. Influence of body temperature on the development of fatigue during prolonged exercise in the heat. $J \mathrm{Appl}$ Physiol 1999: 86(3): 1032-1039.

Haldane JC. The influence of high air temperatures. J Hyg (Lond) 1905: 5(4): 494-513.

Havenith G, Bröde P, den Hartog E, Kuklane K, Holmér I, Rossi RM, Richards M, Farnworth B, Wang, X. Evaporative cooling: effective latent heat of evaporation in relation to evaporation distance from skin. J Appl Physiol 2013: 114(6): 778-785.

Jeukendrup AE. Power output. In: Jeukendrup AE, ed. High Performance Cycling.

Champaign, IL: Human Kinetics, 2002: pp 69-77.

Lind AR. A physiological criterion for setting thermal limits for everyday work. $J$ Appl Physiol 1963: 18(1): 51-56. 
Moran DS, Shitzer A, Pandolf KB. A physiological strain index to evaluate heat stress. American Journal of Physiology 1998: 275 (1 Pt 2): R129-134.

Nagata H. Evaporative heat loss and clothing. J Hum Ergol (Tokyo) 1978: 7(2): 169-175.

Nielsen B. Solar heat load: heat balance during exercise in clothed subjects. Eur J Appl Physiol and Occup Physiol 1990: 60(8): 452-456.

Nielsen M. Regulation der Körpertemperatur bei Muskelarbeit. Skand Arch Physiol 1938: 79(2): 193-230.

Pascoe DD, Shanley LA, Smith EW. Clothing and exercise I: Biophysics of heat transfer between then individual clothing and the environment. Sports Med 1994: 18(1): 38-54.

Ramanathan NL. A new weighting system for mean surface temperature of the human body. J Appl Physiol 1964: 19(3): 531-533.

Saltin B, Hermansen L. Esophageal, rectal, and muscle temperature during exercise. J Appl Physiol 1966: 21(6): 1757-1762.

Saunders AG, Dugas JP, Tucker R, Lambert MI, Noakes TD. The effects of different air velocities on heat storage and body temperature in humans cycling in a hot humid environment. Acta Physiol Scand 2005: 183(3): 241-255. 
Schalader ZJ,Simmons SE, Stannards SR, Mündel T. Exercise modality modulates body temperature regulation during exercise in uncompensable heat stress. Eur J Appl Physiol 2011: 111(5): 757-766.

Tipton MJ. Thermal stress and survival. In: Rainford DJ, Gradwell DP, eds., Ernsting's Aviation Medicine $4^{\text {th }}$ edition. London: Hodder Arnold, 2006: pp213-209.

Vogt S, Schumacher YO, Blum A, Roecker K, Dickhuth HH, Schmid A, Heinrich L. Cycling power output produced during flat and mountain stages in the Giro d'Italia: a case study. $J$ Sports Sci 2007: 25(12): 1299-1305.

Zhang H. Human thermal sensation and comfort in transient and non-uniform thermal environments. PhD thesis, University of California, Berkley. 2003 


\section{Figure Legends}

Figure 1a. Mean rectal temperature during the representative cycle test. Figure 1b. Average mean skin temperature during the representative cycle test. Figure 1c. Average mean body temperature during the representative cycle test. Figure 1d. Mean chest and back skin temperature during the representative cycle test. Letters denote significant difference: $a=$ LOW vs. HI; $b=\mathrm{LOW}$ vs. INT1; $c=\mathrm{LOW}$ vs. INT2; $d=\mathrm{HI}$ vs. INT1; $e=\mathrm{HI}$ vs. INT2; $f=$ INT1 vs. INT2. Bold letters refer to back skin temperature only. Grey shaded areas denote comparison points. Standard deviations are omitted for clarity.

Figure 2. Mean heart rate during the representative cycle test. $a=$ difference LOW vs. HI; $e$ = difference HI vs. INT2. Grey shaded areas denote comparison points. Standard deviations are omitted for clarity

Figure 3. Forehead sweat rates during the representative cycle test. . $a=$ difference LOW vs. HI; $c=$ difference LOW vs. INT2; $d=$ difference HI vs. INT1; $e=$ difference HI vs. INT2. Grey shaded areas denote comparison points. Standard deviations are omitted for clarity. 

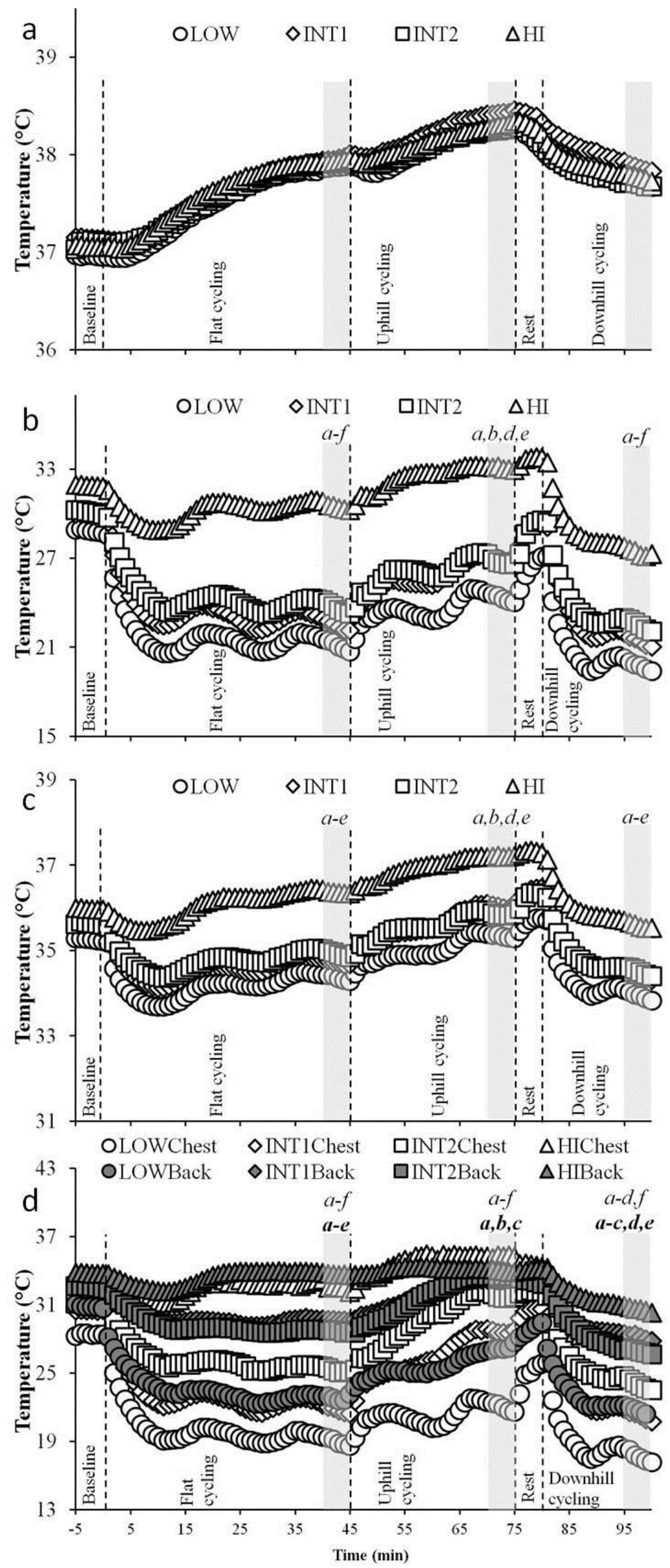

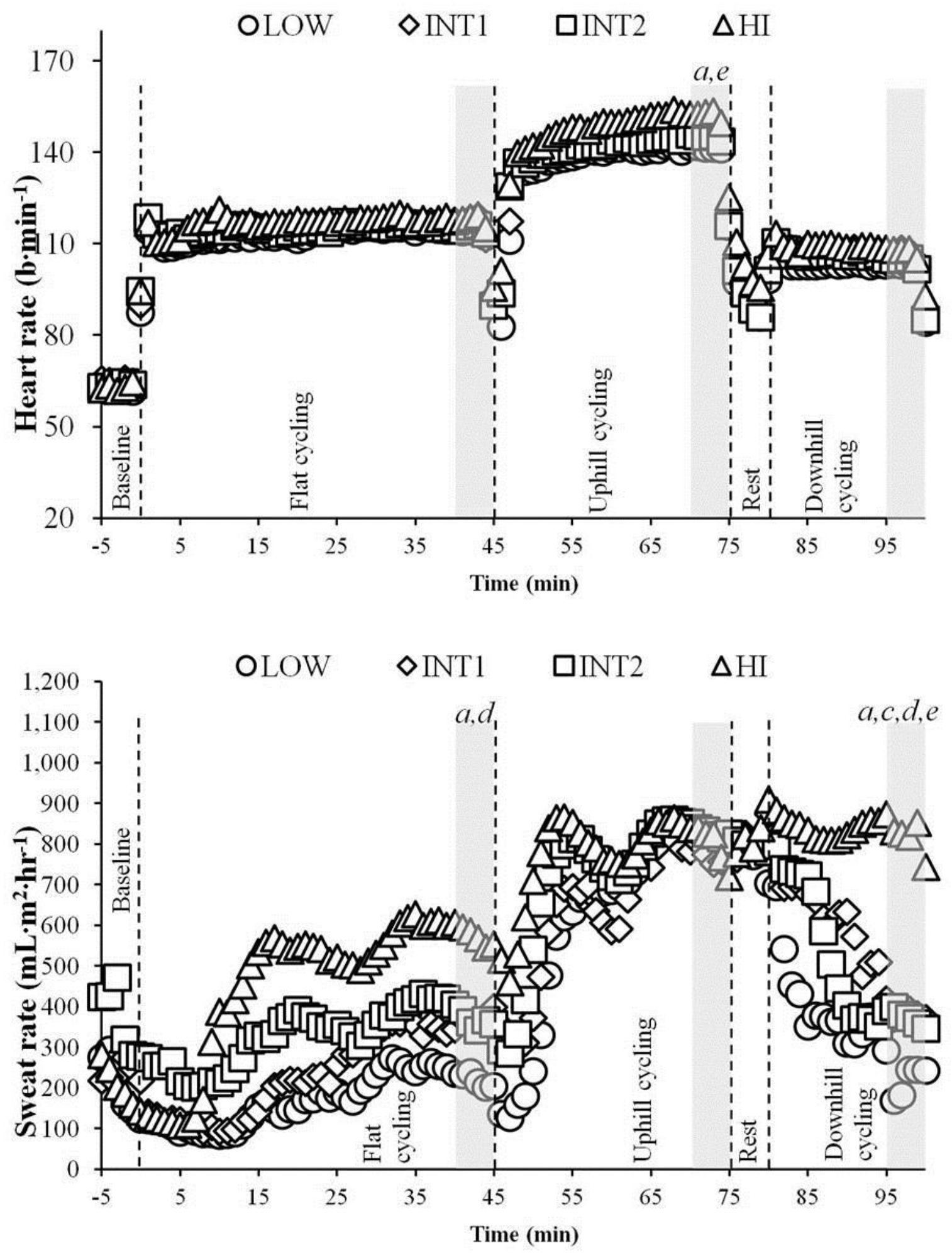